\title{
Erratum: Wong et al (2018)
}

In the article by Wong A, Sanchez-Gonzalez MA, Son W-M, Kwak Y-S, Park S-Y. The effects of a 12-week combined exercise training program on arterial stiffness, vasoactive substances, inflammatory markers, metabolic profile, and body composition in obese adolescent girls. Ped Exerc Sci. 2018;30:480-486. https://doi.org/10.1123/pes.2017-0198, it was incorrectly reported that institutional review board (IRB) approval was obtained. The study was performed in 2011, which predated South Korea's IRB, initiated in 2013. The authors confirm that data collection and all procedures were appropriately performed in accordance with the Declaration of Helsinki, and informed consent from each participant was obtained despite the absence of IRB approval. The authors apologize for this error. 